\title{
Early stage cervical cancer with negative pelvic lymph nodes: morbidity and survival patterns following radical hysterectomy and postoperative adjuvant radiotherapy
}

\author{
Ruchi S. Arora, Shilpa M. Patel*, Ava D. Desai, Meeta M. Makand,
}

Department of Gynaecologic Oncology, Gujarat Cancer and Research Institute, Civil Hospital Campus, Ahmedabad, Gujarat, India

Received: 01 December 2018

Accepted: 29 December 2018

*Correspondence:

Dr. Shilpa M. Patel,

E-mail: drshilpamukesh@gmail.com

Copyright: () the author(s), publisher and licensee Medip Academy. This is an open-access article distributed under the terms of the Creative Commons Attribution Non-Commercial License, which permits unrestricted non-commercial use, distribution, and reproduction in any medium, provided the original work is properly cited.

\section{ABSTRACT}

Background: The objective of the present study was to optimize the role of adjuvant radiotherapy in lymph node negative cervical cancer treated with radical hysterectomy and pelvic lymph node dissection, and to analyse patterns of failure and complication following surgery and radiotherapy in same patients.

Methods: This is retrospective analysis of 98 patients of cancer cervix FIGO Stage IB; IIA treated with radical hysterectomy with bilateral lymphadenectomy and adjuvant radiotherapy between the years 2000 and 2007at our hospital. Among all node negative patients who were operated during this period 97 patients were high risk. [High risk-Two or more of following risk factors: primary tumor size $>4 \mathrm{~cm}$, cervical stromal invasion $\geq 1 / 2$, lymph-vascular space invasion, Unfavorable histology,>].

Results: Median follow up: 79 months [6 months to 109 months], Recurrence rate: $13.26 \%$ [2 Pelvic and 11 distant recurrence] Pelvic control rate: 98 \%Radiotherapy Complications: 4 \% [Grade 3-4].

Conclusions: Adjuvant radiotherapy following radical hysterectomy in high risk node negative early cervical cancer is effective treatment. It reduces pelvic recurrence with acceptable morbidity.

Keywords: Cervical cancer, Negative lymph nodes, Prognostic factors, Radiotherapy, Survival

\section{INTRODUCTION}

Cervical cancer is one of the common malignancies in women worldwide with more than 500,000 new cases diagnosed annually and approximate 250,000 related deaths each year. ${ }^{1}$ Patients with invasive cervical carcinoma limited to the cervix alone [stage 1B] and cervix plus upper vagina [stage 2A] are candidates for radical hysterectomy and pelvic lymphadenectomy. Patients with lymph node metastases have historically been considered high risk, and it is considered strongest predictor of pelvic failure. ${ }^{2,3}$ Patients without lymph node metastases individually have a lower risk of disease recurrence, but still account for half of total early stage cervical cancer recurrence. ${ }^{4}$ Histological type and grade, pathologic tumor size, lymph vascular space invasion and depth of cervical invasion have been identified as poor prognostic factors in patients with negative lymph nodes. ${ }^{4-6}$ The indications for postoperative radiotherapy have varied over the years. Various studies conducted by the American Gynecology Oncology Group (GOG) have provided some clarity regarding the prognostic factors which portend an increased risk of pelvic relapse. The high-risk criteria are positive pelvic nodes, occult parametrial invasion or positive resection lines, for which adjuvant chemoradiation is now a standard 
recommendation. ${ }^{7}$ Patients without these criteria but with combinations of 'intermediate' criteria have varying degrees of risk. The grouping of intermediate-risk factors were done using two criteria (the classic and GOG).

According to the Classic criteria, the intermediate-risk group is defined by the presence of any two factors among three variables (tumour size $>$ or equal to $2 \mathrm{~cm}$, more than one-third stromal invasion, and Lymphovascular space invasion (LVSI)). ${ }^{6,8}$

In contrast, the GOG criteria define the intermediate-risk group more strictly than the Classic criteria, as they include various combinations of the three factors (LVSI, stromal invasion, and tumour size); however, these criteria are too complex and inconvenient, and they demonstrate low sensitivity and are poor predictors of recurrence and survival. 6

The Gynecologic Oncolgy Group Study (GOG) has conducted a prospective randomized study of adjuvant pelvic radiation for patients with these intracervical risk factors.

The study showed the benefit of postoperative radiation in these patients by reducing the recurrence from $28 \%$ in patients who underwent surgery alone to $15 \%$ in patients who received adjuvant radiation. ${ }^{9}$

This retrospective study of lymph node-negative highrisk stage $1 \mathrm{~B}$ and $2 \mathrm{~A}$ cervical cancer patients was undertaken to identify poor prognostic factors and evaluate the effectiveness and sequelae of adjuvant pelvic radiotherapy following radical hysterectomy.

\section{METHODS}

\section{Patient selection}

From January 2000 to December 2007, patients with uterine cervical cancer who completed post-operative adjuvant radiotherapy in at our Institute were taken. 98 patients with negative lymph node metastases but with pathologic prognostic factors predictive of poor outcome [High risk-Two or more of following risk factors: primary tumor size $>4 \mathrm{~cm}$, cervical stromal invasion $\geq 1 / 2$, lymph-vascular space invasion, Unfavorable histology] were evaluated in the study to compare treatment outcome and prognostic factors.

\section{Follow-up}

After completion of radiotherapy patients received regular follow up every 3 months for the first three years with radiological assessment every 6 months and then follow up every 6 months subsequently.

Treatment failure was defined by pathological proof of recurrence or was defined by image study showing regrowth of tumor or enlargement of lymph nodes. A pelvic examination was performed during each follow up. Radiological and/or laboratory investigations were performed when indicated .Patients having blood in stools underwent sigmoidoscopy to identify the cause of bleeding.

\section{Complication analysis}

Rectal and bladder complications and non-rectal gastrointestinal sequelae [small bowel complications] were scored according to RTOG (radiation therapy oncology group) grading scale. Grade3-4 complications were categorized as major complications.

\section{Statistical analysis}

Patient survival was measured from the date of radiotherapy initiation to that of last follow up examination. Survival rate was determined using the Kaplan Meier method.

Long rank tests were used for univariate analysis. Logistic regression analysis was performed for assessment of patient and treatment factors associated with late complications.

\section{RESULTS}

Patient characteristics are

- Mean age- $42 \pm 9.2$ years

- FIGO stage-

- 1B-90

- $2 \mathrm{~A}-8$

- Histological type

- $\quad$ Squamous cell carcinoma-93

- Adenocarcinoma-5

- Tumour size

- $\leq 4 \mathrm{~cm}-71$

- $4 \mathrm{~cm}-27$

- Pathology

- Lymph vascular permeation-14

- Vaginal invasion-8

- More than half thickness-74

Median follow-up-79 months

The five-year disease-free survival [DFS] and overall survival rates [OS] were $85.7 \%$ and $98 \%$ respectively. After 6 months to 109 months of follow up, [median-79 months] 85 patients were alive without evidence of disease, 13 patients died of disease [11 distant and 2 central recurrences]. Median interval from surgery to recurrence was 34 months. The pattern of failure is listed in Table 1. The common site of distant metastasis were Liver [n=2], Spine[n=2], Lung[n=2], Para aortic[n=2], followed by Vulval $[n=1]$, scar $[n=1]$ and umbilical[ $n=1]$. None of the enrolled patients died of other concurrent diseases. 
Table 1: Pattern of failure.

\begin{tabular}{|l|l|l|l|}
\hline $\begin{array}{l}\text { No. of } \\
\text { patients }\end{array}$ & \multicolumn{2}{l}{ Risk factors } & Site of \\
recurrence
\end{tabular}

Overall radiotherapy complication rate was $24 \%$. Grade 1-2 gastrointestinal complications was $6.1 \%$. Patients presented with abdominal discomfort, increased frequency of motion (5-8 times per day), and intermittent bleeding in stool. Grade 3-4 complication rate involving the gastrointestinal tract was $4 \%$.

Table 2: Radiotherapy related complications.

\begin{tabular}{|c|c|c|}
\hline Complications & No. of patients & $\%$ \\
\hline Overall complications rate & $24 / 98$ & 24.4 \\
\hline Percentage needing operation & & 1.0 \\
\hline \multicolumn{3}{|l|}{ Small/large Intestine } \\
\hline Grade $1-2$ & 6 & 6.1 \\
\hline Grade 3-4 & 4 & 4.0 \\
\hline \multicolumn{3}{|l|}{ Bladder } \\
\hline Grade $1-2$ & 4 & 4.0 \\
\hline Grade 3-4 & - & \\
\hline Skin Reaction & 4 & 4.0 \\
\hline Lower leg edema & 3 & 3.0 \\
\hline Obstructive uropathy & 3 & 3.0 \\
\hline
\end{tabular}

Grade 1-2 urinary tract complications was seen in $4 \%$ of patients. Patients presented with either increase frequency of urine or intermittent hematuria. Obstructive uropathy was seen in $3 \%$ of patients. On univariate analysis of prognostic factors age, tumour size, histological type, depth of invasion, grade, vascular permeation, lymphatic permeation and vagina only vascular permeation was found to be significant. ( $\mathrm{p}$ value-0.000).

\section{DISCUSSION}

There is increasing evidence that some subgroups of the patients with Stage 1B-2A lymph node negative cervical carcinoma are at a high risk of recurrence and that the recurrences occur predominantly in the pelvis..$^{3,6}$ Although the survival benefit of adjuvant radiotherapy for lymph node negative cervical cancer is still controversial, optimization of the strategies of adjuvant therapy of these patients should be done. In the current study, all enrolled patients received the same mode of surgical resection and uniform irradiation doses. In present study vascular permeation was independent prognostic factor (Table 3). Previous investigators have identified the independent prognostic value of LVSI in early stage cervical cancer. Burke et al. noted that the presence of LVSI was three times more common in stage 1B cervical carcinoma with negative lymph nodes and clear margins that developed recurrence than in those who did not. ${ }^{4}$ A multicentre GOG study of patients with negative lymph node stage $1 \mathrm{~B}$ squamous cell cervical carcinoma found the disease-free interval to be significantly lower for patients with LVSI. ${ }^{6}$ Vavra et al. reviewed 54 lymph nodes negative stage $1 \mathrm{~B}$ patients with LVSI who underwent radical surgery. Recurrence-free interval was prolonged in those patients with LVSI who received adjuvant radiation, but there was no difference in 5-year survival. ${ }^{10}$ In present study large tumor size, lymph space invasion, deep stromal invasion, worsening differentiation and vaginal involvement were not adverse variable for DFS and OS. 
Table 3: Univariate analysis of prognostic factors.

\begin{tabular}{|c|c|c|c|c|c|}
\hline \multirow{2}{*}{ Variable } & \multirow{2}{*}{ n $(\%)$} & \multicolumn{2}{|c|}{5 years overall survival } & \multicolumn{2}{|c|}{5 years disease free survival } \\
\hline & & $\%$ & p & $\%$ & p \\
\hline \multicolumn{6}{|l|}{ Age } \\
\hline$\leq 40$ & $47(47.96)$ & 97.9 & 0.873 & 85.1 & 0.950 \\
\hline$>40$ & $51(52.04)$ & 98 & & 86.3 & \\
\hline \multicolumn{6}{|l|}{ Histological type } \\
\hline Squamous cell carcinoma & $93(94.89)$ & 97.8 & 0.730 & 84.9 & 0.202 \\
\hline Adeno Carcinoma* & $5(5.11)$ & 100 & & 100 & \\
\hline \multicolumn{6}{|l|}{ Tumor size } \\
\hline$\leq 4 \mathrm{~cm}$ & $71(72.45)$ & 98.6 & 0.514 & 83.1 & 0.430 \\
\hline$>4 \mathrm{~cm}$ & $27(27.55)$ & 96.3 & & 92.6 & \\
\hline \multicolumn{6}{|l|}{ Depth of invasion } \\
\hline$\leq 1 / 2$ & $24(24.49)$ & 95.8 & 0.411 & 91.7 & 0.332 \\
\hline$>1 / 2$ & $74(75.51)$ & 98.6 & & 83.8 & \\
\hline \multicolumn{6}{|l|}{ Nuclear grade } \\
\hline 1 & $6(6.12)$ & 100 & 0.639 & 100 & 0.537 \\
\hline 2 & $74(75.51)$ & 98.6 & & 86.5 & \\
\hline 3 & $17(17.34)$ & 94.1 & & 76.5 & \\
\hline \multicolumn{6}{|c|}{ LP (Lymphatic permeation) } \\
\hline- & $85(86.73)$ & 97.6 & 0.545 & 85.9 & 0.997 \\
\hline+ & $13(13.27)$ & 100 & & 84.6 & \\
\hline \multicolumn{6}{|l|}{ VP (Vascular permeation) } \\
\hline- & 97 (98.98) & 99 & 0.000 & 86.6 & 0.000 \\
\hline+ & $1(1.02)$ & 0.0 & & 0.0 & \\
\hline \multicolumn{6}{|l|}{ Vagina } \\
\hline- & $90(91.83)$ & 97.8 & 0.640 & 86.5 & 0.675 \\
\hline+ & $8(8.17)$ & 100 & & 75 & \\
\hline
\end{tabular}

In addition to nodal involvement, a number of other tumour associated characteristics have been reported to be predictive of outcome by several retrospective studies. Increasing tumor size, deep stromal invasion, lymph vascular space involvement, worsening differentiation, FIGO stage, adverse histology and extension to parametria are all associated with lower 5-year survival rates. ${ }^{3-13}$

Takeshi Kodaira et al in their study reported large tumor size and histology of adenocarcinoma as adverse prognostic factors for DFS and OS. Age, stage, deep stromal invasion, nuclear grade were not adverse prognostic factors. ${ }^{14}$ In present study, bulky disease, full thickness disease, lymphovascular invasion and nuclear grade were surgico-pathological factors present in patients with distant metastasis but were not proved on analysis (Table 1,3).

Takamura et al, reported that 70 patients with lymph node negative, Stage I and II cervical cancer with histologically confirmed parametrial extension received adjuvant standard whole pelvic irradiation to a total dose 50 Gy. Local control was achieved in 66 of 70 patients. However, 28 complications requiring medical treatment occurred and 6 of the 66 patients developed major complications requiring further surgery. ${ }^{15}$ Kridelka et al, reported 25 patients with negative lymph nodes receiving 50.4 Gy of adjuvant small field irradiation to the pelvis. There was one recurrence recorded at 16 months. No major radiation morbidity was reported. 16 Failures in node negative patients usually develop within pelvis but rarely at distant sites. ${ }^{11,12,16}$ Our experiences with adjuvant radiotherapy for patients with lymph node negative cervical cancer achieve compatible pelvic control as with other series .In present study there were 11 distant recurrences and 2 pelvic recurrences [Recurrence rate $13.2 \%$ ].

In studies by Schorge et al and Sartori et al recurrence rate were $20 \%$ and $17 \%$ respectively. ${ }^{11,12}$ Adjuvant radiotherapy probably reduced locoregional recurrences in our series However, since nearly $13.2 \%$ of the patients died of disease; it is justified to intensify the strategy for adjuvant treatment for some subgroups of the patients. Because failures occurred at distant sites, the most feasible approach for the improvement of treatment outcome is a combination of systemic chemotherapy.

In present study Five-year disease-free survival [DFS] and overall survival rates [OS] were $85.7 \%$ and $98 \%$ respectively. Pelvic control rate was $98 \%$. This is comparable with other studies. Similar conclusions were reported by other studies. DFS / OS in study by Takeshi 
Kodaira et al and A Ayan et al were 85.3\%/89.7\% and $87.6 \% / 91 \%$ respectively. ${ }^{13,14}$ Similar Study was done by Sang-Young Ryu et al. Records of 172 patients with any of intermediate factors were taken for analysis. Among 172, 137 patients had two or more intermediate risk factors. There were 12 recurrences, six in the pelvis and six in the distant sites. The classic criteria detected all 12 recurrences; however, GOG criteria could detect only six recurrences.

Although no prognostic factor was significant on multivariate analysis, the difference in the 3-year recurrence-free survival was significant among no further treartment, Radiotherapy and chemoradiotherapy groups. ${ }^{17}$ As has been suggested in recent studies concurrent chemotherapy and radiotherapy may be more effective than radiation alone in terms of distant metastasis and survival. Therefore, introduction of randomized studies is suggested to elucidate the role of chemotherapy for the subgroups of patients. In present study overall radiotherapy complication rate was 24\%.Grade 3-4 complication rate involving the gastrointestinal tract was $4 \%$ (Table-2). Only in one patient ileal resection and anastamosis was done after perforation. Sedlis et al in the GOG 92 trial reported a $2.3 \%$ Grade $3-4$ involving the gastrointestinal tract and $3.1 \%$ involving the genitourinary tract. $^{9}$

The development of distant metastasis could not be reduced through the use of the whole pelvic field: therefore, the benefit of a standard field for this subgroup is doubtful. As radiation morbidity is highly associated with irradiation volume, for the patients with lymph node negative and lymphovascular invasion positive early cervical cancer, authors suggest using a lower pelvic field to minimize gastrointestinal complications. Moreover, Authors advocate the introduction of randomized studies to elucidate the role of chemotherapy for this group of patients.

\section{CONCLUSION}

Postoperative radiotherapy in node-negative stage IB-IIA cervical cancer patients with intermediate risk factors reduced only the incidence of locoregional recurrence. Distant recurrence was the major pattern of treatment failure after adjuvant radiotherapy. In high risk node negative cervical cancer patients most, feasible approach for the improvement of treatment outcome is a combination of systemic chemotherapy.

Funding: No funding sources

Conflict of interest: None declared

Ethical approval: The study was approved by the Institutional Ethics Committee

\section{REFERENCES}

1. Torre LA, Siegel RL, Ward EM, Jemal A. Global cancer incidence and mortality rates and trends--an update. Cancer Epidemiol Biomarkers Prev. 2016; 25(1):16-27.

2. Yeh SA, Leung SW, Wang CJ, Chen HC. Postoperative radiotherapy in early stage carcinoma of the uterine cervix: Treatment results and Prognostic factors. Gynaecol Oncol 1999; 72(1):1015.

3. Lai CH, Hong JH, Hsueh $\mathrm{S}, \mathrm{Ng} \mathrm{KK}$, Chang TC, Tseng CJ, et al. Preoperative prognostic variables and the importance of postoperative adjuvant therapy on the outcomes of stage IB or II cervical patients with or without pelvic lymph node metastasis: an analysis of 891 cases. Cancer. 1999;85(7):1537-46.

4. Peters III WA, Liu PY, Barrett RJ, Stock RJ, Monk BJ, Berek JS, et al. Concurrent chemotherapy and pelvic radiation therapy compared with pelvic radiation therapy alone as adjuvant therapy after radical surgery in high-risk early-stage cancer of the cervix. J Clin Oncol. 2000;18(8):1606-13.

5. Fuller Jr AF, Elliott N, Kosloff C, Hoskins WJ, Lewis Jr JL. Determinants for increased risk of recurrence in patients of the cervix. Gynaecol Oncol 1989;13:33-34.

6. Delgado G, Bundy B, Zaino R, Sevin B, Creasman WT, Major E. Prospective surgical-pathological study of disease-free interval in patients with stage IB squamous cell carcinoma of the cervix: A gynaecologic oncology group study. Gynaecol Oncol 1990;38(3):352-7.

7. Burke TW, Hoskins WJ, Heller PB, Bibro MC, Weiser EB, Park PC. Prognostic factors associated with radical hysterectomy failure .Gynecol Oncol 1980; 26(2):153-9.

8. Samlal RA, van der Velden J, Ten Kate FJ, Schilthuis MS, Hart AA, Lammes FB. Surgical pathological factors that predict recurrence in stage IB and IIA cervical carcinoma patients with negative pelvic lymph nodes. Cancer 1997;80(7):1234-40.

9. Sedlis A, Bundy BN, Rotman MZ, Lentz SS, Munderspach LI, Zaino RJ. A Randomized trial of pelvic radiation therapy versus no further therapy in selected patients with stage $\mathrm{Ib}$ carcinoma of the cervix after radical hysterectomy and pelvic lymphadenectomy: a Gynecologi Oncology Group Study. Gynecol Oncol. 1999;73(2):177-83.

10. Vavra N, Sevelda P, Seifert M, Timar J, Kudielka I, Kucera $\mathrm{H}$, et al. The value of adjuvant irradiation in lymphatic vessel invasion in patients with a cervical carcinoma in histopathological stage Ib and with negative lymph nodes. Strahlenther Onkol.1992; 168(9):524-7.

11. Schorge JO, Molpus KL, Koelliker D, Nikrui N, Goodman A, Fuller AFJr. StageIB and IIA cervical cancer with negative lymph nodes:the role of adjuvant radiotherapy radiotherapy after radical hysterectomy. Gynaecol Oncol. 1997;66(1):31-5.

12. Sartori E, Tisi G, Chiudinelli F, La Face B, Franzini R, Pecorelli S. Early stage cervical cancer: Adjuvant treatment in negative lymph node cases. Gynaecol Oncol 2007;107(1):S170-4. 
13. Ayhan A, Al RA, Baykal C, Demirtas E, Ayhan A, Yüce K. Prognostic factors in FIGO stage IB cervical cancer without Lymph node metastasis and the role of adjuvant radiotherapy after radical hysterectomy. Int J Gynecol Cancer. 2004;14(2):286-292.

14. Kodaira T, Fuwa N, Nakanishi T, Kuzuya K, Sasaoka M, Furutani K et al. Retrospective analysis of postoperative radiotherapy for node negative cervical carcinoma with stage $\mathrm{Ib}-\mathrm{IIb}$ disease. Jap J Clinic Oncol. 2002;32(7):255-61.

15. Takamura A, Mizoe J, Arimoto T, Kamada $\mathrm{T}$, Shirato H, Matsuoka Y, et al . Is postoperative radiotherapy beneficial in the management of stage III squamous cell carcinoma of the uterine cervix with negative nodes and positive parametrial involvement? A retrospective review of 70 patients. Asia Oceania J Obstet and Gynaecol. 1993;19(2): $145-51$
16. Kridelka FJ, Berg DO, Neuman M , Edwards LS, Robertson G, Grant PT, et al. Adjuvant small field pelvic radiation for patients with high risk ,stage IB lymph node negative cervix carcinoma after radical hysterectomy and pelvic lymph node dissection.a pilot study. Cancer 1999;86(10):2059-65.

17. Sang-YoungRyu, Sang-Il Park,Byung-Ho Nam etal Is Adjuvant chemoradiotherapy overtreatment in cervical cancer with intermediate risk factors? Int J Radiation Oncol Biol Phys. 2011;79(3):794-9.

Cite this article as: Arora RS, Patel SM, Desai AD, Makand MM. Early stage cervical cancer with negative pelvic lymph nodes: morbidity and survival patterns following radical hysterectomy and postoperative adjuvant radiotherapy. Int J Reprod Contracept Obstet Gynecol 2019;8:652-7. 